\title{
Coherent Quantum Evolution via Reservoir Driven Holonomies
}

\author{
Angelo Carollo, ${ }^{1}$ Marcelo França Santos, ${ }^{2}$ and Vlatko Vedral ${ }^{3}$ \\ ${ }^{1}$ DAMTP, University of Cambridge, Wilberforce Road, Cambridge CB3 OWA, United Kingdom \\ ${ }^{2}$ School of Physics \& Astronomy, University of Leeds, LS2 9JT, United Kingdom \\ ${ }^{3}$ Departamento de Física, Universidade Federal de Minas Gerais, Belo Horizonte, 30161-970, MG, Brazil
}

(Received 24 July 2005; published 18 January 2006)

We show that in the limit of a strongly interacting environment a system initially prepared in a decoherence-free subspace (DFS) coherently evolves in time, adiabatically following the changes of the DFS. If the reservoir cyclicly evolves in time, the DFS states acquire a holonomy.

DOI: 10.1103/PhysRevLett.96.020403

PACS numbers: 03.65.Vf, 03.65.Yz, 03.67.Pp

Geometric phases and Holonomies are generated by the presence of a curvature (the Berry curvature) in the Hilbert space. The natural way to gain information on this curvature is to perform interferometry on a state vector which has been dragged along closed loops in the Hilbert space. To achieve this task the methods exploited are usually divided into two main categories: in the first one, a state is driven around the space by means of a coherent time evolution [1]. For example, this can be obtained by an adiabatic and cyclic motion, which forces the state to explore eigenspaces of a parameterized Hamiltonian. The second category consists of all those motions that result from non-trace-preserving quantum operations. For example, a series of von Neumann measurements can be used to evolve wave functions by projecting the state vector onto a sequence of overlapping subspaces. As an effect of this measurement-induced motion, a quantum system may indeed acquire a geometric (Abelian or not) phase [2]. The Pancharatnam geometric phase is a typical example of this category $[2,3]$.

Even irreversible quantum processes such as systems interacting with Markovian reservoirs can be used to generate geometric evolutions. Many environmental models have the characteristic of not affecting some particular quantum states when these are lying in suitably "protected" subspaces, so-called decoherence-free subspaces (DFSs) [4]. States lying in these subspaces are stationary; i.e., they do not evolve in time. Having control over the reservoir (e.g., through engineered reservoirs) may imply an indirect control on the protected states of the system [5]. In particular, modifications to the parameters of the reservoir may result in a controlled time evolution of the protected subspace as a whole. If this motion is accomplished in a sufficiently smooth way, it can be shown that states lying in this subspace evolve coherently, thereby acquiring information about the geometry of the space explored.

In this Letter we will explore the possibility of generating (Abelian and non-Abelian) holonomies due to a smooth motion of a DFS in the Hilbert space. It is worth stressing that the existence of a time-dependent DFS is by no means trivial, and its presence often reflects a symmetry preserv- ing evolution. Under a suitable "adiabatic condition" [6], it can be shown that a state lying in a DFS remains inside the subspace and, hence, is rigidly transported around the Hilbert space together with the DFS. The evolution experienced is, in fact, coherent, although entirely controlled via an incoherent phenomenon. When the DFS is eventually brought back to its initial configuration, the net effect is an holonomic transformation on the states lying in this subspace. Notice that the evolution obtained is purely geometrical: no dynamics affects the system inside the DFS; hence, no dynamical phase is accumulated during the evolution.

Moreover, we will show that in the first order correction to the adiabatic evolution the DFS is affected by a decoherence process. This effect is unavoidable, at least conceptually, as far as a nontrivial holonomy is to be obtained. On the other hand, the decoherence time inside the DFS can be made arbitrarily large, in the limit of strong decoherence and/or slow evolution of the subspace. In fact, the decoherence affecting the DFS becomes weaker as the decoherence acting on the outside world gets stronger. This counter-intuitive effect is the essence of the adiabatic approximation. It is, in fact, the fast evolving decoherence process affecting the outside world which decouples the latter from the DFS. Alternatively, this evolution can be understood in terms of a "Zeno effect" [7], where the action of strong environment can be regarded as a measuring apparatus continuously monitoring the DFS of the system. By using this effect in degenerate subspaces it is possible to realize robust holonomic gates for quantum computation, analogous to those realized by means of adiabatic evolutions [8].

Let us first consider a system described by the density operator $\rho$ evolving under the effect of a Markovian environment. The decoherence process due to the interaction with such an environment is described by the following master equation $(\hbar=1)$ :

$$
\frac{d \rho}{d t}=-i[H, \rho]-\sum_{k=1}^{n}\left\{\Gamma_{k}^{\dagger} \Gamma_{k} \rho+\rho \Gamma_{k}^{\dagger} \Gamma_{k}-2 \Gamma_{k} \rho \Gamma_{k}^{\dagger}\right\},
$$


where the commutator generates the coherent part of the evolution and the remaining part represents the effect of the reservoir on the dynamics of the system. The action of each $\Gamma_{k}$ (the Lindblad operators) accounts for the different decohering processes that can affect the system. In the Markovian formalism, a DFS is defined as the common eigenspace of all the Lindblad operators: $\left.\operatorname{Span}\left\{|\psi\rangle\left|\Gamma_{k}\right| \psi\right\rangle=c_{k}|\psi\rangle, \forall k\right\}$.

Suppose that the evolution is solely due to the action of the environment, i.e., the presence of a Hamiltonian evolution can be neglected compared to the effect of the environment $(H=0)$. Consider, now the situation in which the environment depends on some time-dependent external parameter. This time dependence might be regarded as either the action of an experimenter who can control some degree of freedom of the reservoir, or "drift" of some parameter intrinsically occurring in the environment. In general, we will assume the rate of this evolution is very small compared to characteristic time scales of the system. Under this assumption, the evolution is governed by a time-dependent master equation of the form:

$$
\frac{d \rho}{d t}=-\sum_{k}\left\{\Gamma_{k}^{\dagger}(t) \Gamma_{k}(t) \rho+\rho \Gamma_{k}^{\dagger}(t) \Gamma_{k}(t)-2 \Gamma_{k}(t) \rho \Gamma_{k}^{\dagger}(t)\right\},
$$

where the operators $\Gamma_{k}(t)$ are time dependent. For each instant of time we can consider the eigenspaces $\mathcal{K}(t)=$ $\left.\operatorname{Span}\left\{|\psi\rangle\left|\Gamma_{k}(t)\right| \psi\right\rangle=c_{k}|\psi\rangle, \forall k\right\}$ of the instantaneous operators $\Gamma_{k}(t)$. Let $\Pi(t)$ be the projector onto $\mathcal{K}(t)$, and $\Pi_{\perp}=\mathbb{1}-\Pi(t)$ the projector onto the orthogonal complement $\mathcal{K}_{\perp}(t)$. Consider the operator

$$
D(t)=\sum_{k}\left[\Gamma_{k}(t)^{\dagger} \Gamma_{k}(t)-2 c_{k}^{*}(t) \Gamma_{k}(t)+\left|c_{k}(t)\right|^{2} \mathbb{1}\right],
$$

whose Hermitian part $P(t)=\left[D(t)+D^{\dagger}(t)\right] / 2$ is positive semidefinite. The space $\mathcal{K}(t)$ can be defined as the kernel of $D(t)$, i.e., $D(t) \Pi(t)=0$. The assumptions that we will consider are the analogies to the hypothesis of the usual adiabatic theorem [9,10]: (i) $\Pi(t)$ is a chain of projection operators, smoothly depending on time, [more precisely $\Pi(t)$ norm-twice differenciable] [10]; (ii) the nonzero eigenvalues of $P(t)$ are bounded from below by a timeindependent value $\gamma>0$.

Under these assumptions, we will show that: (a) it is possible to formulate an adiabatic limit, for which $\mathcal{K}$ is decoupled from the environmental action, and therefore it is decoherence free; (b) in the adiabatic limit, if the subspace $\mathcal{K}\left(t_{1}\right)$ at some time $t_{1}=t_{0}+T$ coincides with $\mathcal{K}\left(t_{0}\right)$ at the initial time $t_{0}$, the neat evolution experienced by a state belonging to $\mathcal{K}$ is purely geometrical; i.e., the evolution between $t_{0}$ and $t_{1}$ is described by the holonomy (Abelian or not) associated with the path traversed by $\mathcal{K}(t)$ in the Hilbert space; (c) the first order correction to the adiabatic limit is a source of decoherence, whose time scale is inversely proportional to the smallest time scale of the decoherence affecting the $\mathcal{K}_{\perp}$, the latter being the orthogonal complement of $\mathcal{K}$.

We would like to describe the evolution at some time $t_{1}=t_{0}+T$ of states initially prepared inside the subspace $\mathcal{K}\left(t_{0}\right)$, in the limit in which the time rate of motion of $\mathcal{K}(t)$ is much smaller than the smallest characteristic time scale of the system, which is $\gamma^{-1}$. It is then convenient to introduce a time-independent parameter $s=\left(t-t_{0}\right) / T$, with $s \in[0,1]$, and solve the master equation in the limit of $T \rightarrow \infty$. By following a similar approach of the standard adiabatic theorem [9], let us consider a unitary operator $O(s)$ for which: $\frac{d}{d s}\left(O^{\dagger} \Pi O\right)=0$ with $O(0)=\mathbb{1}$. The operator fulfilling this condition is defined by the equation $i \frac{d}{d s} O(s)=G(s) O(s)$ [with initial condition $O^{\dagger}(0)=\mathbb{1}$ ] $[9,10]$ where the generator is $G=i\left[\frac{d \Pi}{d s}, \Pi\right]+Q(s)$, and $Q(s)$ is an arbitrary block diagonal Hermitian operator, i.e., $\Pi Q(s) \Pi_{\perp}=0$. By definition, $O^{\dagger}$ describes the change of picture to the "rotating frame," which rigidly follows the subspace $\mathcal{K}(s)$. In fact, in this picture, the projector $\bar{\Pi}(s) \equiv O^{\dagger}(s) \Pi(s) O^{\dagger}(s)^{\dagger}=\Pi(0)$, and the corresponding subspace $\bar{K}$ is time independent. The freedom in the choice of the generator $G(s)$ corresponds to the arbitrary choice of a smooth chain of basis within $\mathcal{K}$ and $\mathcal{K}_{\perp}$, and it is often referred in this context as gauge freedom. Under reparametrization $t \rightarrow s=\left(t-t_{0}\right) / T$, and change of frame $\rho \rightarrow$ $\bar{\rho}=O^{\dagger} \rho O$, Eq. (2) takes the form:

$$
\frac{d \bar{\rho}}{d s}=i[\bar{G}, \bar{\rho}]-\gamma T\left\{\bar{D}(s) \bar{\rho}+\bar{\rho} \bar{D}^{\dagger}(s)-2 \sum_{k} \bar{\Gamma}_{k} \bar{\rho} \bar{\Gamma}_{k}^{\dagger}\right\}
$$

with $\bar{G}=O^{\dagger} G O, \bar{\Gamma}_{k}=\left(O^{\dagger} \Gamma_{k} O-c_{k} \mathbb{1}\right) / \sqrt{\gamma}$, and $\bar{D}=$ $O^{\dagger} D O / \gamma$. Notice that $\bar{\Gamma}_{k} \Pi=0$ and that the operator $\bar{D}$ has been renormalized, so that its Hermitian part $\bar{P}=$ $\left(\bar{D}+\bar{D}^{\dagger}\right) / 2=\sum_{k} \bar{\Gamma}_{k}^{\dagger} \bar{\Gamma}_{k}$ has a minimum no-null eigenvalue not smaller than 1 .

From Eq. (4) it is possible to see that the incoherent part of the evolution affects only system states lying outside the subspace $\overline{\mathcal{K}}$. However, this does not prevent a state initially prepared in $\overline{\mathcal{K}}$ to be exposed to decoherence. Indeed, the off diagonal terms $\bar{G}_{\text {off }} \equiv \bar{\Pi}_{\perp} \bar{G} \bar{\Pi}$ and $\bar{G}_{\text {off }}^{\dagger}$ of $\bar{G}$ can couple the subspace $\overline{\mathcal{K}}$ to its complementary, and may eventually spoil the coherence of the former. In order to unwind the effect of the perturbation introduced by $G$ from the main decoherence process, it is convenient to introduce a transformation which allows the evolution to be described in terms of two effectively decoupled manifolds. To this end, consider the following effective non-Hermitian Hamiltonian:

$$
\tilde{H}(s) \equiv-e^{i S(s)}[\eta \bar{G}(s)+i \bar{D}(s)] e^{-i S(s)}
$$

where $\eta \equiv 1 / \gamma T$ is the adiabatic parameter and $S(s)$ is a (non-Hermitian) operator defined by the condition that: $(\alpha) \bar{\Pi} \tilde{H} \bar{\Pi}_{\perp}=\bar{\Pi}_{\perp} \tilde{H} \bar{\Pi}=0$ (with $\bar{\Pi}_{\perp}=\mathbb{1}-\bar{\Pi}$ ) and ( $\beta) \bar{\Pi} S \bar{\Pi}=\bar{\Pi}_{\perp} S \bar{\Pi}_{\perp}=0$ [11]. Let us now analyze the time evolution of the density matrix under the transforma- 
tion $e^{i S}$. To this end consider the operator $\tilde{\rho}=e^{i S} \bar{\rho} e^{-i S^{\dagger}}$. Notice that although this transformation is not unitary, $\tilde{\rho}$ is still a positive semidefinite operator, and hence it is a valid density matrix, up to a normalization factor $N=$ $\operatorname{Tr}\left(\bar{\rho} e^{i\left(S-S^{\dagger}\right)}\right)>0$. By assuming for simplicity that the time derivative of $S$ is negligible, the evolution of $\tilde{\rho}$ is given by

$$
\frac{d \tilde{\rho}}{d s} \simeq-\frac{i}{\eta}\left[\tilde{H} \tilde{\rho}-\tilde{\rho} \tilde{H}^{\dagger}\right]+\frac{2}{\eta} \sum_{k} \tilde{\Gamma}_{k} \tilde{\rho} \tilde{\Gamma}_{k}^{\dagger},
$$

with $\tilde{\Gamma}_{k} \equiv e^{i S} \bar{\Gamma}_{k} e^{-i S}$. The advantage of this expression is that $\tilde{H}$ is now block diagonal and the main coupling effects are only due to the second term. We are interested in the evolution of the system in the limit of $\eta \ll 1$. It is, then, convenient to consider the expansion of $S$ in series of $\eta$ : $S=\eta S_{1}+\eta^{2} S_{2}+O\left(\eta^{3}\right)$, and, correspondingly, the expansion of the effective Hamiltonian $\tilde{H}=\tilde{H}_{0}+\eta \tilde{H}_{1}+$ $\eta^{2} \tilde{H}_{2}+O\left(\eta^{3}\right)$. By using the above conditions $(\alpha)$ and $(\beta)$ it is possible to show that:

$$
\begin{gathered}
\tilde{H}_{0}=-i \bar{D}(s), \\
\tilde{H}_{1}=-\bar{\Pi} \bar{G} \bar{\Pi}-\bar{\Pi}_{\perp} \bar{G} \bar{\Pi}_{\perp}, \\
\tilde{H}_{2}=-\frac{1}{2} \bar{\Pi}\left[i S_{1}, \bar{G}\right] \bar{\Pi}-\frac{1}{2} \bar{\Pi}_{\perp}\left[i S_{1}, \bar{G}\right] \bar{\Pi}_{\perp},
\end{gathered}
$$

where $S_{1}=\bar{D}^{-1} \bar{G}_{\text {off }}-$ H.c.. Notice that $\bar{D}^{-1}$ in the last expression is well defined, as it is restricted to the subspace $\mathcal{K}_{\perp}$ where $D$ is invertible. In the limit of $\eta \ll 1$ the evolution of $\tilde{\rho}$ can be expressed as follows:

$$
\frac{d \tilde{\rho}}{d s} \simeq \frac{1}{\eta} \mathcal{L}_{-1}[\tilde{\rho}]+\mathcal{L}_{0}[\tilde{\rho}]+\eta \mathcal{L}_{1}[\tilde{\rho}]+O\left(\eta^{2}\right) .
$$

The largest contribution in (10) is given by

$$
\mathcal{L}_{-1}[\tilde{\rho}]=-\sum_{k}\left\{\bar{\Gamma}_{k}^{\dagger} \bar{\Gamma}_{k} \tilde{\rho}+\tilde{\rho} \bar{\Gamma}_{k}^{\dagger} \bar{\Gamma}_{k}-2 \bar{\Gamma}_{k} \tilde{\rho} \bar{\Gamma}_{k}^{\dagger}\right\},
$$

where we also expressed $\tilde{\Gamma}_{k}=\bar{\Gamma}_{k}+i \eta\left[S_{1}, \bar{\Gamma}_{k}\right]+O\left(\eta^{2}\right)$ and retained only the zeroth contributions in $\eta$. By definition of $\bar{\Gamma}_{k}$ Eq. (11) acts trivially on the partial density operator $\bar{\rho}_{D F}=\bar{\Pi} \tilde{\rho} \bar{\Pi}$, i.e., $\mathcal{L}_{-1}\left[\bar{\rho}_{D F}\right]=0$. Therefore, the most relevant nontrivial evolution affecting the state $\bar{\rho}_{D F}$ is given by the term $\mathcal{L}_{0}$ in the adiabatic expansion:

$$
\mathcal{L}_{0}\left[\bar{\rho}_{D F}\right]=i\left[\bar{G}_{D F}, \bar{\rho}_{D F}\right] \quad \text { with } \quad \bar{G}_{D F}=\bar{\Pi} \bar{G} \bar{\Pi},
$$

where the fact that $\bar{\Gamma}_{k} \bar{\rho}_{D F}=0$ has been used. This expression explicitly demonstrates that retaining only the terms up to the zeroth order in the adiabatic parameter $\eta$ yields to an evolution for $\bar{\rho}_{D F}$ which is unitary, and, therefore, coherent and trace preserving. This shows the existence of an adiabatic limit for $\eta \rightarrow 0$, in which the evolution is confined-by the decoherence process itself-into a suitable subspace, in which the evolution maintains its coherence. It is worth noticing that, due to the nonunitary transformation $e^{i S}$, the new decoherencefree subspace $\check{\mathcal{K}}$ is slightly modified from the original $\overline{\mathcal{K}}$. Indeed, the presence of the generator of the adiabatic motion, $G$, deforms the DFS, whose (generalized) projectors are to be identified with $\bar{\Pi} \rightarrow \check{\Pi}=e^{i S} \bar{\Pi} e^{-i S^{\dagger}} \simeq \bar{\Pi}+$ $i \eta\left\{S_{1}, \bar{\Pi}\right\}$. As expected, in the limit $\eta \rightarrow 0, \check{\mathcal{K}}$ converges to $\overline{\mathcal{K}}$.

The evolution of the partial density matrix $\bar{\rho}_{D F}$ can be easily solved in the adiabatic limit and formally expressed as

$$
\begin{aligned}
\bar{\rho} & =U(s) \rho(0) U^{\dagger}(s) \\
\text { with } \quad U(s) & =\mathcal{P} \exp \left(i \int_{0}^{s} \bar{G}_{D F}(\tau) d \tau\right),
\end{aligned}
$$

where $\mathcal{P}$ is a path ordering operator. Let us now consider a closed evolution of $\mathcal{K}$, i.e., an evolution for which $\Pi\left(t_{1}\right)=\Pi\left(t_{0}\right)$. Then it is possible to express the total evolution as

$$
U(1)=\mathcal{P} \exp \left(i \int_{0}^{1} \bar{G}_{D F}(\tau) d \tau\right)=\mathcal{P} \exp (\oint \vec{A} d \vec{\lambda}),
$$

where the right hand side expresses the time evolution in terms of a path integral. The operator $\vec{A}$, is a (non-Abelian) holonomic connection defined as a vector of components

$$
A_{\lambda_{i}}=-\bar{\Pi} O^{\dagger} \frac{\partial O\left(\lambda_{1}, \lambda_{2} \ldots\right)}{\partial \lambda_{i}} \bar{\Pi},
$$

where $\lambda$ 's are a set of variables parameterizing the transformations $O(s)$. $U(1)$ has an inherently geometrical nature: it is independent of the time rate and only a function of the structure of the underlying Hilbert space. The nontrivial value of $U(1)$ is, in fact, a manifestation of the curvature of the Hilbert space experienced by $\tilde{\rho}$ when it is dragged along in the subspace $\check{\mathcal{K}}$. The connection $\vec{A}$ behaves as a proper gauge potential: under a change of basis in $\mathcal{K}, O(s) \rightarrow O^{\prime}(s)=O(s) \Omega(s)$, where $[\bar{\Pi}, \Omega]=$ $0, \vec{A}$ transforms as $\vec{A} \rightarrow \vec{A}^{\prime}=\Omega^{-1} \vec{A} \Omega+\Omega^{-1} \nabla \Omega$. By choosing a convenient gauge for which $\Pi O^{\prime}(1) \Pi=\Pi$, it is possible to show that the net effect on the state $\rho \simeq$ $\tilde{\rho}_{D F}$ after a complete cyclic evolution is given by

$$
U^{\prime}(1)=\mathcal{P} \exp \oint i\left[\frac{d \Pi}{d s}, \Pi\right] d s,
$$

which is independent of the gauge chosen [12]. Equation (12) implies that the largest relevant noncoherent contribution to the dynamics of $\tilde{\rho}_{D F}$ can originate only at a further order in the adiabatic parameter $\eta$, i.e., from $\mathcal{L}_{-1}$. By substituting Eq. (9) in Eq. (6), and by making use again of the fact that $\bar{\Gamma}_{k} \bar{\rho}_{D F}=0$, it is possible to show that the first order correction to an evolution restricted to $\check{\mathcal{K}}$ can be given in the following closed form:

$$
\begin{aligned}
\mathcal{L}_{1}\left[\bar{\rho}_{D F}\right]= & -i\left[Z, \bar{\rho}_{D F}\right]-\sum_{k}\left\{\Lambda_{k}^{\dagger} \Lambda_{k} \bar{\rho}_{D F}+\bar{\rho}_{D F} \Lambda_{k}^{\dagger} \Lambda_{k}\right. \\
& \left.-2 \Lambda_{k} \bar{\rho}_{D F} \Lambda_{k}^{\dagger}\right\},
\end{aligned}
$$

where $\quad \Lambda_{k} \equiv \bar{\Gamma}_{k} S_{1}=\bar{\Gamma}_{k} \bar{D}^{-1} G_{\text {off }} \quad$ and $\quad Z \equiv S_{1}^{\dagger} R S_{1}=$ $i G_{\text {off }}^{\dagger}\left(\bar{D}^{-1 \dagger}-\bar{D}^{-1}\right) G_{\text {off }}$. The superoperator $\mathcal{L}_{1}$ generates 
an incoherent evolution with a typical time scale on the order of $\eta^{-1}$. It is worth stressing that, although Eq. (15) looks similar to a master equation in the Lindblad form, it generates an evolution that, when restricted to the subspace $\breve{\mathcal{K}}$, can be non-trace-preserving. This is, in fact, the case when $\Pi_{\perp} \Lambda_{k} \Pi \neq 0$. As, by construction, the evolution in the whole Hilbert space is trace preserving, this clearly indicates a leakage of population from the DSF to the orthogonal subspace, with a time rate on the order $\eta^{-1}$.

Remarkably, since $\mathcal{L}_{1}$ is the largest order incoherent term affecting the subspace $\check{\mathcal{K}}$ and it is proportional to $\gamma^{-1}$, it implies a decoherence time in $\breve{\mathcal{K}}$ which is inversely proportional to the original decoherence time affecting the complementary space: a stronger decoherence in $\check{\mathcal{K}}_{\perp}$ implies weaker environmental effects in $\breve{\mathcal{K}}$. This apparently counter-intuitive phenomenon is the essence of the adiabatic approximation. It is the fast dynamics associated with the incoherent processes acting on $\check{\mathcal{K}}_{\perp}$ which is responsible for decoupling the subspace $\breve{\mathcal{K}}$ from its complement. This fast evolution averages out the effect of $G_{\text {off }}$ and results in a decoherence time scale in $\breve{\mathcal{K}}$ which is quadratic in $T$. This is the reason why, in spite of the relatively long time scale $T$ needed for the adiabatic approximation, the system in $\check{\mathcal{K}}$ is guaranteed to be coherent for a time which scales with a further order of magnitude in $T$. Alternatively, the same result can be interpreted from the perspective of a quantum Zeno effect. The decoupling between $\breve{\mathcal{K}}$ and $\breve{\mathcal{K}}_{\perp}$ can be regarded as the effect of a continuous measurement process performed by the environment. The latter continuously monitors whether the system state leaks to $\check{\mathcal{K}}_{\perp}$ and projects it back into $\overline{\mathcal{K}}$ in a time scale of $\gamma^{-1}$, which is much faster than the leakage process induced by $G_{\text {off }}$.

On the light of the previous discussion on $\mathcal{L}_{1}$, a remark on the generator of the holonomy is in order. Notice that Eq. (14) implies that, after a closed motion, a nontrivial net evolution is achieved only if $i\left[\frac{d \Pi}{d s}, \Pi\right]=G_{\text {off }}+G_{\text {off }}^{\dagger}$ is nonvanishing. Indeed $\left[\frac{d \Pi}{d s}, \Pi\right]=0$ implies $d \Pi / d s=0$, which yields to a trivial motion of the subspace $\mathcal{K}$, and the resulting holonomy has to be trivial. This is in agreement with the idea that the holonomy is the result of the curvature of the Hilbert space experienced by the subspace $\mathcal{K}(t)$ as the latter traverses a nontrivial closed motion. Therefore, for an holonomic evolution in $\overline{\mathcal{K}}$, the latter subspace needs to be coupled to $\overline{\mathcal{K}}_{\perp}$ via $G_{\text {off }}$, which, as seen in Eq. (15), exposes $\tilde{\rho}_{0}$ to environmental effects. This implies that decoherence on $\overline{\mathcal{K}}$ is in principle unavoidable, as far as a nontrivial holonomy is concerned. On the other hand, as seen before, $\mathcal{L}_{1}$ appears as a small perturbation in the adiabatic expansion, and therefore its effects are adiabatically eliminated in the limit of large enough $T$.

The results presented in this manuscript reinforce the idea that geometric evolutions not only do not depend on the dynamical procedures used to generate them, but can even be obtained in the presence of strong coupling to external reservoirs (nonunitary evolutions). As shown here, the interaction with the environment can be used to adiabatically manipulate quantum states in a coherent way, and generate holonomies in arbitrary dimensional DFS. Apart from their fundamental interest, these results, combined with techniques for reservoir engineering, may provide alternative approach for robust quantum computation. As for any other geometrical procedure, this holonomic evolution has an intrinsically fault tolerant nature [13], and due its very nature it is free from dynamical contributions, which are known to be generally more sensitive to errors.

We acknowledge the support of EU, under TOPQIP project. M. F. S. acknowledges the support of CNPq. V. V. acknowledges also support from EPSRC and the British Council in Austria.

[1] M. V. Berry, Proc. R. Soc. A 392, 45 (1984).

[2] Y. Aharonov and M. Vardi, Phys. Rev. D 21, 2235 (1980); G. Cassinelli et al., Phys. Rev. A 49, 3229 (1994); P. Facchi et al., Phys. Lett. A 257, 232 (1999); J. Anandan and A. Pines, Phys. Lett. A 141, 335 (1989).

[3] S. Pancharatnam, Proc. Indian Acad. Sci. A 44, 247 (1956).

[4] G. M. Palma, K.-A. Suominen, and A. K. Ekert, Proc. R. Soc. A 452, 567 (1996); L.-M. Duan and G.-C. Guo, Phys. Rev. Lett. 79, 1953 (1997); P. Zanardi and M. Rasetti, Phys. Rev. Lett. 79, 3306 (1997); D. A. Lidar, I. L. Chuang, and K. B.Whaley, Phys. Rev. Lett. 81, 2594 (1998); A. Barenco et al., SIAM J. Comput. 26, 1541 (1997); L. Viola et al., Science 293, 2059 (2001).

[5] A. R. R. Carvalho et al., Phys. Rev. Lett. 86, 4988 (2001).

[6] For a general study of the adiabatic approximation in open systems, see M. S. Sarandy and D. A. Lidar, Phys. Rev. A 71, 012331 (2005).

[7] B. Misra and C. M. Sudarshan, J. Math. Phys. (N.Y.) 18, 756 (1977); P. Facchi and S. Pascazio, Phys. Rev. Lett. 89, 080401 (2002).

[8] R. G. Unanyan, B. W. Shore, and K. Bergmann, Phys. Rev. A 59, 2910 (1999); L. M. Duan, J. I. Cirac, and P. Zoller, Science 292, 1695 (2001).

[9] A. Messiah, Quantum Mechanics (North-Holland, Amsterdam, 1962); T. Kato, J. Phys. Soc. Jpn. 5, 435 (1950).

[10] G. Nenciu, J. Phys. A 13, L15 (1980).

[11] Such an operator $S(\eta)$ differentiable around $\eta=0$ (where $S(0)=\mathbb{1})$ exists; see N. Ghosh et al., SIAM J. Numer. Anal. 34, 1255 (1997) and references therein.

[12] It is worth analyzing this result under the perspective of "virtual subspaces;" see P. Zanardi, Phys. Rev. Lett. 87, 077901 (2001); [See also M. Nordling and E. Sjoqvist, Phys. Rev. A 71, 012110 (2005)].

[13] G. De Chiara and G. M. Palma, Phys. Rev. Lett. 91, 090404 (2003); P. Solinas, P. Zanardi, and N. Zanghì, Phys. Rev. A 70, 042316 (2004); A. Carollo et al., Phys. Rev. Lett. 90, 160402 (2003). 